\title{
Identification of key endometrial microRNAs and its target genes associated with pathogenesis of recurrent implantation failure by integrated bioinformatics analysis
}

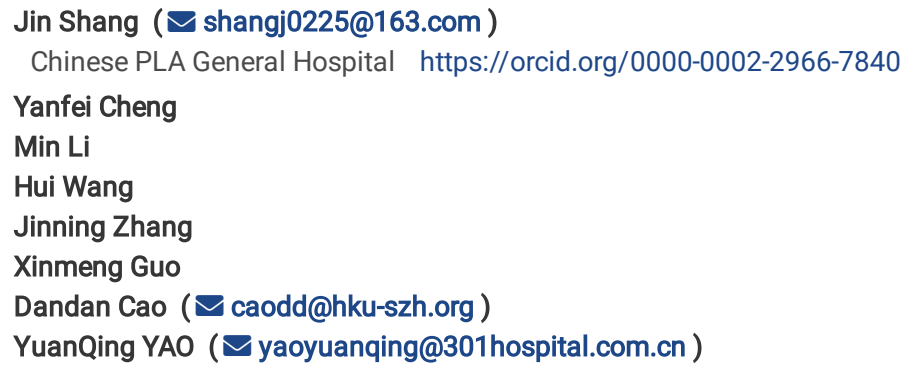

\section{Research Article}

Keywords: recurrent implantation failure, differentially expressed genes, differentially expressed miRNAs, bioinformatic.

Posted Date: January 28th, 2022

DOI: https://doi.org/10.21203/rs.3.rs-1257766/v1

License: (c) (i) This work is licensed under a Creative Commons Attribution 4.0 International License. Read Full License 


\section{Abstract \\ Purpose}

Recurrent implantation failure (RIF) is an enormous challenge for In Vitro Fertilization (IVF) clinicians. Understanding of the molecular mechanisms of RIF helps to predict prognosis and develop new therapeutic strategies. The study is designed to identify diagnosis biomarkers for RIF, as well as the potential mechanisms underlying RIF by utilizing public databases together with experimental validation.

\section{Methods}

Two microarray datasets of RIF patients and healthy controls endometrium were downloaded from the Gene Expression Omnibus (GEO) database. Firstly, differentially expressed microRNAs(miRNAs) (DEMs) were identified and their target genes were predicted. Then we identified differentially expressed genes (DEGs) and selected hub genes through Protein-protein interaction (PPI) analyses. Functional enrichment analyses of DEGs and DEMs were conducted. Furthermore, the key DEMs which targeted those hub genes were selected to obtain key miRNA-target genes network. The key genes in miRNA-target genes network were validation by a single cell RNA-sequencing dataset of endometrium from GEO. Finally, we selected two miRNA-target genes pairs to further experimental validation used dual-luciferase assay and quantitative PCR (qPCR).

\section{Results}

We identified 49 DEMs between RIF patients and fertile group and found 136,678 target genes. Then 325 DEGs were totally used to construct PPI network, and 33 hub genes were selected. 25 DEMs targeted 16 key DEGs were obtained to establish key miRNA-target genes network, and 16 key DEGs were validated by single cell RNA-sequencing dataset. Finally, the target relationship of hsa-miR-199a-5p-PDPN and hsa-miR-4306-PAX2 were verified by dual-luciferase assay, and there were significant differences in the expression of those genes between RIF and fertile group by PCR $(P<0.05)$.

\section{Conclusion}

We constructed miRNA-target gene regulatory networks associated with RIF which provide new insights regarding the underlying pathogenesis of RIF. hsa-miR199a-5p-PDPN, hsa-miR-4306-PAX2 could be further explored as potential biomarkers for RIF, and their detection in endometrium could be applied in clinics to estimate the probability of successful embryo transfer.

\section{Introduction}

Assisted reproductive technology (ART) has made a breakthrough in the history of modern science. Many infertile couples are benefited from the ART treatment, but a significant portion of them are still frustrated following multiple failed attempts, which lead to the emergence of a new challenge: recurrent implantation failure (RIF) [1].

RIF does not have a universal definition. The preimplantation genetic diagnosis consortium of the European Society of Human Reproduction and Embryology Preimplantation Genetic Diagnosis Consortium has defined RIF as $>3$ failed embryo transfers with high-quality embryos or the failed transfer of $\geq 10$ embryos in multiple transfers, which was widely accepted[2]. The aetiology of RIF is complex and is not attributed to a single abnormality as a successful implantation involved numbers of components [3]. Traditionally, implantation has been considered as a process mainly involving the embryo and the endometrium, combined with other factors, such as cumulus cell competency[4]. The contribution of embryo factors to RIF can be partly ruled out by performing preimplantation genetic testing for aneuploidy to select euploidy embryos for transfer [5]. However, inadequate uterine receptivity is considered to be responsible for nearly $2 / 3$ of implantation failures [6] [7].

The only time for embryo implantation is window of implantation (WOI), when endometrium enters a narrow window of a receptive state during the midsecretory phase of menstrual cycle [8]. Many complex morphological and functional changes occur in the endometrial WOI during embryo implantation [9]. RIF is associated with molecular and functional changes of the endometrium receptivity in the WOI. Current studies of RIF patients endometrium receptivity often based on the transcriptomic signature, and the large number of molecules have been proposed as receptive biomarkers[10]. However, these huge numbers of biomarkers sometimes present differences among individuals which bring misleading judgments on the fertility status[11]. So it is urgent to find some credible key expressed genes in endometrium to evaluate the endometrial receptivity. Meanwhile, microRNA (miRNAs) have been widely reported to be involved in the function of endometrium as transcriptional regulators of gene expression during embryo implantation[12]. Studies found that the target genes of some miRNAs were involved in cyclic remodelling of the endometrium, including endometrial maturation to the receptive state, and might contribute to an adhesive interaction at the cell surface[13].

To provide insight into the potential roles of miRNAs and their target genes causing RIF, we analysed two public microarray datasets from the Gene Expression Omnibus (GEO) database, GSE71332 (miRNA) [14] and GSE111974 (mRNA) [15], and performed integrated bioinformatics analyses to investigate the potential endometrial causes of RIF. Differentially expressed miRNAs (DEMs) and their predicted target genes were firstly identified between RIF and fertile control. Then we identified expressed genes (DEGs) from which some key genes were obtained that constructed miRNA-target gene regulatory networks to explore the molecular mechanisms underlying RIF. The schematic illustration of this study is shown in Figure 1. Each step was elaborated in the following subsections. 


\section{Methods And Materials Ethics Statements and Clinical Subjects}

This study was approved by the Ethics Committee of the General Hospital of the People's Liberation Army. Participants were recruited from the First Affiliated Hospital of the People's Liberation Army. Ten patients who failed to become pregnant after $\geq 3$ embryo transfers were included in the RIF group. In the control group, six patients who conceived after their first embryo transfer or had a previous gestation history were recruited. Endometrial specimens were collected at the mid-luteal phase of a menstrual cycle, and the specimens were placed in $3 \mathrm{ml}$ of Sample Preservation buffer (Yikon, China) and stored at $-80^{\circ} \mathrm{C}$ refrigerator until use.

\section{Microarray Dataset Collection}

To identify the key microRNAs of RIF, we downloaded the raw data of microarray datasets using the keywords "recurrent implantation failure" combined with "microRNA" to search for genome-wide expression studies in the Gene Expression Omnibus (GEO) database from National Centre for Biotechnology Information (NCBI). The microRNA dataset GSE71332 [16] and the gene expression dataset GSE111974 [15] were generated using the GPL18402 (Agilent046064 Unrestricted_Human_miRNA_V19.0_Microarray) and the GPL17077 (Agilent-039494 SurePrint G3 Human GE v2 8×60K Microarray 039381)platforms, respectively. GSE71332 contained endometrial samples collected at the WOI ( $\mathrm{LH}+5$ to LH+9) from seven RIF patients and five normal fertile women, while the endometrial samples collected at WOI (LH+7 to LH+10) from 24 RIF patients and 24 fertile controls were included in GSE111974. Detailed information of two datasets is shown in Table 1.

\section{Differential Gene Expression Analysis}

Series Matrix Files of GSE71332 were downloaded and imported into the R-studio (Version 1.2.1335), and raw data of GSE111974 were downloaded from the GEO into R-studio, as well. The robust multi-array average (RMA) method was used for data prepressing [17, 18]. The non-human genes probes from the chips were removed, and multiple probes related to the same gene were reduced to one and summarised as median values. MRNA microarray probes were annotated using the annotated files in the platform GPL18402 while removing probes that did not match any gene symbols.

DEMs and DEGs were identified by the R Bioconductor package 'limma' (version 4.0.3) [19] by comparing the RIF and the healthy control groups. For DEM and DEG identification, $|\log 2 \mathrm{FC}|>1$ and adjusted $\mathrm{P}<0.05$, were regarded as statistically significant.

\section{Functional and Pathway Enrichment Analysis of DEMs and DEGs}

Gene Ontology (GO) terms of biological processes, cellular component and molecular functions were used to determine the function of the DEMs and DEGs. Kyoto Encyclopaedia of Genes and Genomes (KEGG) pathway analysis was used to explore the significant pathways of DEGs. We performed GO enrichment analysis for DEMs using an open access, standalone functional enrichment tool FunRich (http://www.funrich.org) [20]. For DEGs, we used the R package clusterProfiler package (version 3.18.1) [21], and KEGG pathway analyses were performed using the Cytoscape (Version: 3.8.2) with the ClueGo (Version: 2.5.7) and the CluePedia (Version: 1.5.7) plug-in. The p-values were all less than 0.05 which were calculated using a two-sided hypergeometric test and Benjamini-Hochberg adjustment.

\section{Protein-protein Interactions Network Construction and Selection of Hub Genes}

Protein-protein interactions (PPIs) of DEGs were screened using the STRING database [22] (Version: 11.0), with confidence threshold value $\geq 0.4$ (medium confidence). Networks were visualised using the Cytoscape (version 3.8.2, https://www.cytoscape.org/). The CytoNCA plugin [23] (Version 2.1.6) was used to analyse network topology properties for nodes, and the score of gene nodes was calculated using three centrality methods (degree centrality, betweenness centrality, and closeness centrality) [24-26]. In addition, a Cytoscape-plugin Molecular Complex Detection (MCODE) [27] (Version: 2.0.0,

http://apps.cytoscape.org/apps/MCODE) was adopted to select the key modules of the PPI network with degree cut-off $=2$, max. depth $=100$, node score cutoff $=0.2$, and $k$-Core $=2$. Several top nodes with high degrees based on the three centrality methods and key modules of the PPI network were identified as hub genes for further analysis.

\section{Integrative analysis of DEMs and DEGs}

We predicted the potential target genes of the DEMs from GSE71332 using the miRWalk [28] (Version: 3.0, https://mirwalk.umm.uni-heidelberg.de) a miRNAmRNA binding sites prediction tool [29]. Based on the typical inhibitory effect of a miRNA on its downstream target, only upregulated miRNA-downregulated mRNA and downregulated miRNA-upregulated mRNA pairs were identified by checking the direction of expression change of predicted miRNA-target pairs in DEMs and DEGs. The regulatory network of the identified DEM-DEG pairs was constructed and visualised using the Cytoscape (version 3.8.2). Moreover, network topology was analysed to identify key miRNA-target gene pairs.

\section{Expression analysis of key genes in Single cell RNA-sequencing dataset of endometrium samples}

To identify the key genes demonstrating variation during the whole menstrual cycle between control and RIF samples, the raw data of a single-cell RNA-seq transcriptome profile GSE111976 [30] was downloaded from the NCBI GEO database. The GSE111976 dataset covers the single-cell transcriptome profile of endometrium biopsies sampled from 19 healthy and fertile females, at 4-27 days of their menstrual cycle, which can be used to systematically characterize endometrial transformation across the healthy human menstrual cycle in preparation for embryo implantation. We identified the expression of the key genes at the mid- and late-secretory stages in six cell types: stromal fibroblasts, unciliated endothelial cells, ciliated endothelial cells, macrophages, and lymphocytes. The expression of key genes was analysed in the four stages. 


\section{Verification of selected key gene expression and its upstream miRNA in clinical samples}

Total RNA was extracted from endometrial cells using the RNA Quick Purification kit (ES science, China). The determination of RNA concentration and purity was performed using a spectrophotometer (Nanodrop 2000, Thermo Scientific). Total RNA with good purity and integrity was reverse-transcribed using the Fast All-in-One RT Kit (ES science, China) and the Mir-X miRNA First-Strand Synthesis kit (Takara, Japan), respectively. Then, cDNA samples were stored at $-80^{\circ} \mathrm{C}$. Real-time qPCR was performed to determine the key miRNA-target gene pairs expression levels. miRNA expression was measured using the SYBR $B$ Green kit (Takara, Japan) with an ABI StepOne PCR instrument. U6 was used as an internal control for miRNA and GAPDH was used as an internal control for mRNA. Relative quantification of gene expression was performed using the comparative $\mathrm{CT}\left(2^{-} \triangle \triangle \mathrm{CT}\right)$ method.

\section{Validation of miRNA-target relationship by Dual-luciferase reporter assay}

The 3'-untranslated regions (UTRs) of the target genes were amplified by PCR, using the genomic DNA of 293T cells as a template and the designed amplification primers (Table 2). Both wild-type and mutated forms of the UTRs for the genes were synthesized. After cloning the amplified WT3'UTR (wild-type vector) or MUT 3'UTR (mutant vector) into the pmir-GLO vector, validation of the reporter assay was performed with the dual luciferase reporter system (Promega E1910, USA). For detection of relative fluorescence values, an miRNA mimic or non-target control was respectively co-transfected into 293T cells withWT3'UTR pmir-GLO-Reporter or MUT 3'UTR pmir-GLO-Reporter. The luciferase fluorescence was measured by SpectraMax®i3 (Molecular Devices, USA).

\section{Statistical analysis}

We used the Graphpad Prism software (Version 8.0.1) for statistical analysis. Data are expressed as mean \pm standard error. Differences were analysed using the Student's t-test. Statistical significance was set at $\mathrm{P}<0.05$.

Results

\section{Identification of Differentially Expressed miRNAs in RIF}

A total of 49 DEMs (42 up-regulated and 7 down-regulated) were identified between the RIF and the control groups, and volcano plots with their corresponding $\log 2 \mathrm{FC}$ values are shown in Figure 2.

\section{Predicting Target Genes of DEMs and Functional Enrichment Analysis}

Online tools miRWalk (Version 2.0) based on 6 bioinformatic, utilized to predict the potential candidate target genes of aberrant miRNAs, such as algorithms miRWalk2.0, Pictar2, PITA, RNA22v2, RNAhybrid2.1, and Targetscan6.2. Only the target genes which were common in the prediction of all the above algorithms were screened out. Finally, a total of 136,678 target genes for 49 DEMs were obtained.

In order to get insight into the functions and mechanisms of these DEMs, biological process (BP), cellular component (CC), and molecular function (MF) were enriched in GO terms. The most significantly enriched GO terms in the in the BP-associated category was Signal transduction. In the CC category, 49 miRNAs were suggested to be related to the Nucleus and Cytoplasm. In the MF category, DEMs were mainly involved in Transcription factor activity (Figure 3 ).

\section{Identification of RIF Differentially Expressed Genes and Functional Enrichment Analysis}

Compared with the RIF patients and fertile control groups in GSE111974 datasets by R Bioconductor package 'limma' (version 4.0.3), 325 DEGs (200 upregulated and 125 down-regulated) were identified for further analysis (Figure 4A).

In order to have an in-depth understanding of biological significance of these DEGs, we performed GO enrichment by R package clusterProfiler package and KEGG enrichment using the Cytoscape software with ClueGo and CluePedia plug-in (Figure 4B). In GO enrichment analysis, the BP-associated category was significantly enriched in Carboxylic acid transport and Organic acid transport. The identified DEGs were significantly enriched in the Apical part of cell and Apical plasma membrane in the CC category. While in the MF category, the DEGs were mainly enriched in Endopeptidase inhibitor activity. The most significantly enriched KEGG terms of the DEGs included arrhythmogenic right ventricular cardiomyopathy (ARVC), TNF signalling pathway, vascular smooth muscle contraction, cell adhesion molecules (CAMs), purine metabolism, pyrimidine metabolism, NF-kappa B signalling pathway, arachidonic acid metabolism, phospholipase D signalling pathway, AMPK signalling pathway, longevity regulating pathway, adipocytokine signalling pathway, glucagon signalling pathway, and insulin resistance (Figure 4C).

\section{DEGs Prioritization by PPI Network Analysis and Key Target Genes Selection}

To investigate the interactions of DEGs, we constructed the PPI network for the 325 DEGs. The PPI network contained 229 nodes and 373 interaction pairs (Figure 5A). A total of 11 key cluster modules were screened out by the MCODE plugin. Figure 5B and 5C show the first two key modules of the PPI network; one contained eight nodes and 28 interaction pairs, and the other with nine nodes and 30 interaction pairs. The CytoNCA was used for the network topology analysis based on the three centrality methods (degree centrality, betweenness centrality, and closeness centrality). Table 3 shows the top 20 nodes with high degrees of network topology analysis. Then 11 up-regulated genes and 22 down-regulated genes were screened as hub genes, such as lysophosphatidic acid receptor 3 ( $L P A R 3)$, angiotensinogen (AGT), and podoplanin (PDPM).

A total of 16 hub genes from PPI network analysis were identified, which had predicted target relationship with those DEMs (Figure 6). As a result, we regarded these 16 genes (LPAR3, PAX2, PDE6A, TSC2, AGT, ASS, GJA1, GNG4, GPR68, KDR, NKX2-1, PDPN, PENK, PROM1, RRM2, VCAM1), including four up-regulated and 12 down-regulated, as key genes in our analysis (Table 4), 


\section{Single cell RNA-sequencing dataset Analysis of the prioritized targets genes}

The expression levels of the key genes in normal endometrium was examined in a single-cell sequencing dataset, which divides the normal female menstrual cycle into four phases (menstrual and early-proliferative phase, late-proliferative phases, early-secretory phase, mid and late-secretory phase). As shown in Figure 7A, most of the key genes were distinctly expressed in stromal fibroblasts and unciliated epithelial cells during the mid- and late-secretory phase. The expressions of some of the key genes such as GJA1, PROM1, ASS1, and PDPN were high, while that of the LPAR3, PDE6A, and PAX2 were low in this mid- and late-secretory phase. We selected four representative genes, GJA1, PDPN, $L P A R 3$, and $P A X 2$, to observe their change in expression among the four menstrual phases (Figure 7B). PDPNand GJA1 exhibited upregulation when entering the mid-secretory stage, whereas the expression of $\angle P A R 3$ and $P A X 2$ gradually decreased from the late-proliferative phases to the mid and late-secretory phases. Interestingly in GSE111974, the expressions of PDPN and GJA1 were downregulated in RIF patients in the window of receptivity of endometrium when compared with that of healthy people, whereas that of $L P A R 3$ and $P A X 2$ were upregulated in RIF patients. In the mid-secretory phase, the endometrium prepares for embryo attachment. Therefore, these results indicated that dysregulation of PDPN, GJA1, LPAR3, and PAX2 expression in RIF patients might disturb endometrial receptivity and affected embryo implantation.

\section{Experimental Validation of the Significant miRNA-target genes pairs}

Two miRNA-target genes pairs were selected for further experimental validation, due to their function in uterine or adnexa in existing reports that were related to RIF. The expression and regulatory relationships between hsa-miR-4306 with PAX2, hsa-miR-199a-5p with PDPN were confirmed. RIF patients and matched control were recruited for endometrium collection. No significant differences were observed between the two groups in endometrial stages, age, and body mass index $(P<0.05)$. The expression of candidate miRNAs and their proposed targets were examined in the collected endometrium. As shown in Figure $8 A-B$, $P D P N$ and hsa-miR-4306 were down-regulated in the endometrial cells of RIF patients compared to that in non-RIF individuals $(P<0.05)$, whereas $P A X 2$ and hsa-miR-199a-5p were up-regulated in the endometrium samples of RIF $(P<0.05)$. These results were consistent with that found from the microarray data. Moreover, we found a negative expression correlation between hsa-miR-4306 and PAX2, as well as between hsa-miR-199a-5p and PDPN, consistent with an inhibitory role of miRNAs on their targets.

To validate the direct targeting relationship between the suggested miRNAs and mRNA, we performed a dual-luciferase assay. Both wild type and mutated 3'UTRs (containing the predicted target sites) luciferase reporter plasmids for PDPN and PAX2 were constructed. The results showed that overexpression of hsa-miR-199a-5p and hsa-miR-4306 could significantly inhibit the relative luciferase activity of PDPN-WT3'UTR and PAX2-WT3'UTR (P = 0.0007 for hsa-miR199a-5p and $P D P N ; \mathrm{P}=0.0177$ for hsa-miR-4306 and $P A X 2$ respectively, whereas the effects were abolished with the mutated $3^{\prime} U T R$ s. validating the direct regulatory role of hsa-miR-199a-5p on PDPN expression, and hsa-miR-4306 on PAX2 expression (Figure 8C).

\section{Discussion}

MiRNAs are evolutionarily conserved small non-coding RNAs that control post-transcriptional gene expression by targeting mRNAs for degradation and translational repression [31, 32]. MiRNAs exert their activity by binding to the 3'-UTR of the target mRNAs and a single miRNA can regulate multiple target mRNAs simultaneously [33]. Increasing evidence suggests that miRNAs play a key role in the regulation of a range of fundamental cellular processes; thus, abnormal regulation of miRNAs is associated with diseases, including RIF [34-36].

Herein, our study identified 49 DEMs from the miRNA dataset and 325 DEGs from the mRNA expression profiling dataset, consisting of 42 up-regulated and 7 down-regulated miRNAs, as well as 200 up-regulated genes and 125 down-regulated genes in RIF sample tissues compared with healthy endometrial tissues. Enrichment analysis were conducted among those DEMs and DEGs, indicating that these genes were related to the functions of vasoconstriction, regulation of blood vessel diameter and regulation of systemic arterial blood pressure and to the pathways such as cell adhesion molecular (CAM) pathway. And a recent study showed a significant reduction in the secretion of mid-term endometrial CAMs in women with RIF, which may be responsible for embryo implantation failure [37].

Then the top 33 hub genes with high degrees of three network topology analysis were identified by PPI included 11 up-regulated genes and 22 down-regulated genes; some of which have previously been reported to be related to recurrent implantation failure. The prostaglandin production by cyclooxygenase 2(COX2, also known as PTGS2) plays an important role in endometrial receptivity and embryo implantation [38]. KDR (also known as VEGFR2) is a hub gene in the PPI network and is overexpressed in RIF compared to fertile samples. Liu et al. [39] used ultrasound molecular imaging to examine the expression of VEGFR2 on the uterine vascular endothelium for evaluation of endometrial receptivity. Furthermore, the hub module was significantly enriched in the renin-angiotensin system (RAS)-associated genes such as AGT, AVP, and AVPR1A. In a previous study, AVP receptors was predominantly expressed in non-pregnant women [40], which is consistent with our finding that AVPR1A was up-regulated in RIF samples, whereas Pringle et al. found that dysregulation of endometrial RAS could be a predisposing factor to endometrial cancer [41]. Specifically, they examined the prevalence of RAS-related single nucleotide polymorphisms and showed that $A G T$ levels were less prevalent in women with endometrial cancer than in controls, and $A G T$ caused removal of angiotensin I, both are anti-angiogenic factors. Therefore, investigation should be made to determine if repression of RAS in in the endometrium would influence angiogenesis during embryo implantation and lead to RIF.

When constructing a miRNA-target gene regulatory network based on DEMs and DEGs, we found 4 down-regulated and 19 up-regulated key miRNAs in the network. Among these, hsa-miR-1260b is known to mediate growth differentiation factor 11 (GDF11)-Smad-dependent signaling, an important regulatory mechanism for the proliferation of vascular smooth muscle cells (VSMCs), which is regulated by hypoxia [42]. What's more, in the regulatory networks, hsamiR-135b-5p regulated two key down-regulated genes: PDPN and AGT. Overexpression of PDPN, a small mucin-like type-1 transmembrane protein and a specific marker for lymph vessel endothelial cells [43], significantly increased endothelial cell adhesion, migration, and tube formation, whereas inhibition of PDPN expression decreased dermal lymphatic endothelial cell adhesion [44]. PDPN is a specific marker for the lymphatic endothelium in histopathology [45].

Page 5/17 
Hsa-miR-135b-5p in serum exosome promotes proliferation and migration of VSMCs [46] and could be a prognostic biomarker in breast cancer and lupus nephritis $[47,48]$. Suppression of hsa-miR-135b-5p promotes the decidualisation of the endometrial stroma and ameliorates the decidualisation of human ESCs (hESCs) from RIF patients [49]. Consistently, our study found that has-miR-135b-5p was significantly up-regulated in the RIF samples. Has-miR-135b-5p has been implicated in the decidualisation mechanism of hESCs. For instance, its target gene activating transcription factor 3 ( $A F T 3$ ) promotes embryo adhesion in vitro by transcriptionally increasing leukaemia inhibitory factor expression in epithelial cells [50], and knockdown of this AFT3 significantly disrupts structural features of decidualized stromal cells [51]. Previous research has also shown that placental growth factor promotes trophoblast cell invasion by up-regulating ATF3 expression and down-regulating hsa-miR-199a-5p expression [52], which was overexpressed and target on PDPN in our study. Hsa-miR-199a-5p stimulates ovarian granulosa cell apoptosis in polycystic ovary syndrome, and its downregulation promotes ovarian granulosa cell viability and inhibits apoptosis, while induces an increase in serum estradiol (E2) levels and a decrease in serum anti-mullerian hormone levels, luteinizing hormone, and follicle-stimulating hormone levels in a polycystic ovarian syndrome rat model [53]. Moreover, hsa-miR-199a-5p is suggested to play important roles in many cancers such as ovarian cancer, non-small cell lung cancer, and laryngeal cancer in humans [54-56]. Another miRNA that targets PAX2, is hsa-miR4306. It is a new therapeutic target for triple-negative breast cancer, which is transcriptionally regulated by oestrogen receptor-alpha, human epidermal growth factor receptor 2, and progesterone receptor [57]. In addition, the intercellular transfer of hsa-miR-4306 by platelet microparticles inhibit the migration of human monocyte-derived macrophages through the VEGFA/ERK1/2/NF-KB signalling pathway, which may influence the immunological environment at the maternal-foetal interface [58]. And its target gene PAX2 acts as a tumour suppressor in endometrial carcinogenesis [59], which is an accurate marker of precancerous endometrial hyperplasia [60]. Reduction of PAX2 expression in murine oviductal cells enhances estrogen receptor signaling [61].

Above all, we choose the two miRNA-target gene pairs hsa-miR-4306-PAX2 and hsa-miR-199a-5p-PDPN for further experimental validation. We verified their different expressed in endometrium between RIF patients and fertile control by qPCR, and their target relationship by dual-luciferase assay. These potential regulatory patterns may contribute to the discovery of new molecular targets for the diagnosis of RIF.

There are limitations in the present study. Firstly, the sample sizes of the two microarray datasets above are small, especially for the miRNA profile dataset. Therefore, future studies with larger sample sizes are required. Secondly, the results require further investigation and validation both in vitro and in vivo. Notably, further mechanistic studies of the RIF miRNA - target gene regulatory network could provide insight into the molecular mechanisms of RIF. Furthermore, the detection of these expression of miRNAs and key genes in endometrium could be helpful to predict the probability of successful transplantation in clinics. The outcome could help clinicians achieve risk assessment of RIF before IVF treatment, and help choose the best treatment strategy during oocyte retrieval and embryo transfer, in order to obtain enough embryos available for transfer when faced with RIF and achieve a satisfactory clinical outcome.

\section{Declarations}

\section{Data Availability Statement}

The gene expression of RIF in our study were obtained from GEO (https://www.ncbi.nlm.nih.gov/geo).

\section{Acknowledgements}

We appreciate the contributions of Dr. Ze Zhang to provide technical guidance and assistance.

\section{Author Contributions}

Jin Shang: Protocol/project development, data analysis and manuscript writing

Yanfei Cheng: Protocol/project development and data management

Min Li: Manuscript editing and data collection

Hui Wang: Data collection

Jinning Zhang: Data collection

Guoxin Meng: Data collection

Dandan Cao: Data analysis and manuscript editing

Yuanqing Yao: Protocol development

\section{Funding}

This work was supported by Key Technologies Research and Development Program of China (Grant No.2018YFC1003100)

\section{Conflict of Interest:}

The authors declare that they have no conflict of interest.

\section{References}


1. Coughlan C, Ledger W, Wang Q, Liu F, Demirol A, Gurgan T, Cutting R, Ong K, Sallam H, Li TC (2014) Recurrent implantation failure: definition and management. Reprod Biomed Online 28(1):14-38

2. Ata B, Kalafat E, Somigliana E (2021) A new definition of recurrent implantation failure on the basis of anticipated blastocyst aneuploidy rates across female age. Fertil Steril 116(5):1320-1327

3. Bashiri A, Halper KI, Orvieto R (2018) : Recurrent Implantation Failure-update overview on etiology, diagnosis, treatment and future directions Reproductive biology and endocrinology: RB\&E 16(1):121

4. Benkhalifa M, Demirol A, Sari T, Balashova E, Tsouroupaki M, Giakoumakis Y, Gurgan T (2012) Autologous embryo-cumulus cells co-culture and blastocyst transfer in repeated implantation failures: a collaborative prospective randomized study. Zygote (Cambridge, England) 20(2):173-180

5. Kimelman D, Pavone ME (2021) Non-invasive prenatal testing in the context of IVF and PGT-A. Best Pract Res Clin Obstet Gynaecol 70:51-62

6. Melford SE, Taylor AH, Konje JC (2014) Of mice and (wo)men: factors influencing successful implantation including endocannabinoids. Hum Reprod Update 20(3):415-428

7. Craciunas L, Gallos I, Chu J, Bourne T, Quenby S, Brosens JJ, Coomarasamy A (2019) Conventional and modern markers of endometrial receptivity: a systematic review and meta-analysis. Hum Reprod Update 25(2):202-223

8. Wang W, Vilella F, Alama P, Moreno I, Mignardi M, Isakova A, Pan W, Simon C, Quake SR (2020) Single-cell transcriptomic atlas of the human endometrium during the menstrual cycle. Nat Med 26(10):1644-1653

9. von Grothusen C, Lalitkumar S, Boggavarapu NR, Gemzell-Danielsson K, Lalitkumar PG (2014) Recent advances in understanding endometrial receptivity: molecular basis and clinical applications. Am J Reprod Immunol 72(2):148-157

10. Wang X, Yu Q (2018) An update on the progress of transcriptomic profiles of human endometrial receptivity. Biol Reprod 98(4):440-448

11. Coutifaris C, Myers ER, Guzick DS, Diamond MP, Carson SA, Legro RS, McGovern PG, Schlaff WD, Carr BR, Steinkampf MP et al (2004) Histological dating of timed endometrial biopsy tissue is not related to fertility status. Fertil Steril 82(5):1264-1272

12. Liang J, Wang S, Wang Z (2017) Role of microRNAs in embryo implantation. Reproductive biology and endocrinology: RB\&E 15(1):90

13. Kang Y-J, Lees M, Matthews LC, Kimber SJ, Forbes K, Aplin JD (2015) MiR-145 suppresses embryo-epithelial juxtacrine communication at implantation by modulating maternal IGF1R. J Cell Sci 128(4):804-814

14. Shi C, Shen H, Fan LJ, Guan J, Zheng XB, Chen X, Liang R, Zhang XW, Cui QH, Sun KK et al (2017) Endometrial MicroRNA Signature during the Window of Implantation Changed in Patients with Repeated Implantation Failure. Chin Med J (Engl) 130(5):566-573

15. Bastu E, Demiral I, Gunel T, Ulgen E, Gumusoglu E, Hosseini MK, Sezerman U, Buyru F, Yeh J (2019) Potential Marker Pathways in the Endometrium That May Cause Recurrent Implantation Failure. Reprod Sci 26(7):879-890

16. Shi C, Shen H, Fan L-J, Guan J, Zheng X-B, Chen X, Liang R, Zhang X-W, Cui Q-H, Sun K-K et al (2017) Endometrial MicroRNA Signature during the Window of Implantation Changed in Patients with Repeated Implantation Failure. Chin Med J (Engl) 130(5):566-573

17. Bolstad BM, Irizarry RA, Astrand M, Speed TP (2003) A comparison of normalization methods for high density oligonucleotide array data based on variance and bias. Bioinformatics 19(2):185-193

18. Irizarry RA, Hobbs B, Collin F, Beazer-Barclay YD, Antonellis KJ, Scherf U, Speed TP (2003) Exploration, normalization, and summaries of high density oligonucleotide array probe level data. Biostatistics 4(2):249-264

19. Ritchie ME, Phipson B, Wu D, Hu Y, Law CW, Shi W, Smyth GK (2015) limma powers differential expression analyses for RNA-sequencing and microarray studies. Nucleic Acids Res 43(7):e47

20. Pathan M, Keerthikumar S, Ang CS, Gangoda L, Quek CY, Williamson NA, Mouradov D, Sieber OM, Simpson RJ, Salim A et al (2015) FunRich: An open access standalone functional enrichment and interaction network analysis tool. Proteomics 15(15):2597-2601

21. Yu G, Wang L-G, Han Y, He Q-Y (2012) clusterProfiler: an R package for comparing biological themes among gene clusters. OMICS 16(5):284-287

22. Szklarczyk D, Gable AL, Lyon D, Junge A, Wyder S, Huerta-Cepas J, Simonovic M, Doncheva NT, Morris JH, Bork P et al (2019) STRING v11: protein-protein association networks with increased coverage, supporting functional discovery in genome-wide experimental datasets. Nucleic Acids Res 47(D1):D607D613

23. Tang Y, Li M, Wang J, Pan Y, Wu F-X (2015) CytoNCA: a cytoscape plugin for centrality analysis and evaluation of protein interaction networks. BioSystems 127:67-72

24. Cukierski WJ, Foran DJ (2008) Using Betweenness Centrality to Identify Manifold Shortcuts. Proc IEEE Int Conf Data Min 2008:949-958

25. Du Y, Gao C, Chen X, Hu Y, Sadiq R, Deng Y (2015) A new closeness centrality measure via effective distance in complex networks. Chaos 25(3):033112

26. Opsahl T, Agneessens F, Skvoretz JJSn (2010) Node centrality in weighted networks: Generalizing degree and shortest paths. 32:245-2513

27. Bader GD, Hogue CWV (2003) An automated method for finding molecular complexes in large protein interaction networks. BMC Bioinformatics 4:2

28. Sticht C, De La Torre C, Parveen A, Gretz N (2018) miRWalk: An online resource for prediction of microRNA binding sites. PLoS ONE 13(10):e0206239

29. Ding J, Li X, Hu H (2016) TarPmiR: a new approach for microRNA target site prediction. Bioinformatics 32(18):2768-2775

30. Wang W, Vilella F, Alama P, Moreno I, Mignardi M, Isakova A, Pan W, Simon C, Quake SR (2020) Single-cell transcriptomic atlas of the human endometrium during the menstrual cycle. Nat Med 26(10):1644-1653

31. Olena AF, Patton JG (2010) Genomic organization of microRNAs. J Cell Physiol 222(3):540-545

32. Lim LP, Lau NC, Garrett-Engele P, Grimson A, Schelter JM, Castle J, Bartel DP, Linsley PS, Johnson JM (2005) Microarray analysis shows that some microRNAs downregulate large numbers of target mRNAs. Nature 433(7027):769-773

33. Santamaria X, Taylor H (2014) MicroRNA and gynecological reproductive diseases. Fertil Steril 101(6):1545-1551

Page $7 / 17$ 
34. Catalanotto C, Cogoni C, Zardo G (2016) : MicroRNA in Control of Gene Expression: An Overview of Nuclear Functions. International journal of molecular sciences $17(10)$

35. Liang J, Wang S, Wang Z (2017) Role of microRNAs in embryo implantation. Reproductive biology and endocrinology: RB\&E 15(1):90

36. Tan Q, Shi S, Liang J, Zhang X, Cao D, Wang Z (2020) : MicroRNAs in Small Extracellular Vesicles Indicate Successful Embryo Implantation during Early Pregnancy.Cells $9(3)$

37. Guo F, Si C, Zhou M, Wang J, Zhang D, Leung PCK, Xu B, Zhang A (2018) Decreased PECAM1-mediated TGF- $\beta 1$ expression in the mid-secretory endometrium in women with recurrent implantation failure. Hum Reprod 33(5):832-843

38. Cha J, Sun X, Dey SK (2012) Mechanisms of implantation: strategies for successful pregnancy. Nat Med 18(12):1754-1767

39. Liu H, Chen Y, Yan F, Han X, Wu J, Liu X, Zheng H (2015) : Ultrasound molecular imaging of vascular endothelial growth factor receptor 2 expression for endometrial receptivity evaluation. Theranostics 5(2):206-217

40. Fuchs AR, Behrens O, Maschek H, Kupsch E, Einspanier A (1998) Oxytocin and vasopressin receptors in human and uterine myomas during menstrual cycle and early pregnancy. Hum Reprod Update 4(5):594-604

41. Pringle KG, Delforce SJ, Wang Y, Ashton KA, Proietto A, Otton G, Blackwell CC, Scott RJ, Lumbers ER (2016) Renin-angiotensin system gene polymorphisms and endometrial cancer. Endocrine connections 5(3):128-135

42. Seong M, Kang H (2020) : Hypoxia-induced miR-1260b regulates vascular smooth muscle cell proliferation by targeting GDF11. BMB reports 53(4):206211

43. Quintanilla M, Montero-Montero L, Renart J, Martín-Villar E (2019) : Podoplanin in Inflammation and Cancer.International journal of molecular sciences20(3)

44. Bieniasz-Krzywiec P, Martín-Pérez R, Ehling M, García-Caballero M, Pinioti S, Pretto S, Kroes R, Aldeni C, Di Matteo M, Prenen H et al (2019) : PodoplaninExpressing Macrophages Promote Lymphangiogenesis and Lymphoinvasion in Breast Cancer.Cell Metab30(5)

45. Hamada M, Ebihara Y, Nagata K, Yano M, Kogashiwa Y, Nakahira M, Sugasawa M, Nagatsuka H, Yasuda M (2020) Podoplanin is an efficient predictor of neck lymph node metastasis in tongue squamous cell carcinoma with low tumor budding grade. Oncol Lett 19(4):2602-2608

46. Zhang M, Chen D, Zhang F, Zhang G, Wang Y, Zhang Q, He W, Wang H, Chen P (2020) Serum exosomal hsa-miR-135b-5p serves as a potential diagnostic biomarker in steroid-induced osteonecrosis of femoral head. American journal of translational research 12(5):2136-2154

47. Bao C, Lu Y, Chen J, Chen D, Lou W, Ding B, Xu L, Fan W (2019) Exploring specific prognostic biomarkers in triple-negative breast cancer. Cell Death Dis 10(11):807

48. Garcia-Vives E, Solé C, Moliné T, Vidal M, Agraz I, Ordi-Ros J, Cortés-Hernández J (2020) : The Urinary Exosomal miRNA Expression Profile is Predictive of Clinical Response in Lupus Nephritis. International journal of molecular sciences21(4)

49. Wang Z, Liu Y, Liu J, Kong N, Jiang Y, Jiang R, Zhen X, Zhou J, Li C, Sun H et al (2021) ATF3 deficiency impairs the proliferative-secretory phase transition and decidualization in RIF patients. Cell Death Dis 12(4):387

50. Cheng X, Liu J, Shan H, Sun L, Huang C, Yan Q, Jiang R, Ding L, Jiang Y, Zhou J et al (2017) : Activating transcription factor 3 promotes embryo attachment via up-regulation of leukemia inhibitory factor in vitro. Reproductive biology and endocrinology: RB\&E 15(1):42

51. Wang F, Li J, Wang H, Zhang F, Gao J (2020) Activating transcription factor 3 inhibits endometrial carcinoma aggressiveness via JunB suppression. Int J Oncol 57(3):707-720

52. Mei Z, Huang B, Zhang Y, Qian X, Mo Y, Deng N (2019) Histone deacetylase 6 negatively regulated microRNA-199a-5p induces the occurrence of preeclampsia by targeting VEGFA in vitro. Biomed Pharmacother 114:108805

53. Shao S, Wang H, Shao W, Liu N (2020) miR-199a-5p stimulates ovarian granulosa cell apoptosis in polycystic ovary syndrome. J Mol Endocrinol 65(4):187-201

54. Hua Q, Jin M, Mi B, Xu F, Li T, Zhao L, Liu J, Huang G (2019) LINC01123, a c-Myc-activated long non-coding RNA, promotes proliferation and aerobic glycolysis of non-small cell lung cancer through miR-199a-5p/c-Myc axis. J Hematol Oncol 12(1):91

55. Gan X, Zhu H, Jiang X, Obiegbusi SC, Yong M, Long X, Hu J (2020) : CircMUC16 promotes autophagy of epithelial ovarian cancer via interaction with ATG13 and miR-199a. Mol Cancer 19(1):45

56. Li DJ, Wang X, Yin WH, Niu K, Zhu W, Fang N (2020) MiR-199a-5p suppresses proliferation and invasion of human laryngeal cancer cells. Eur Rev Med Pharmacol Sci 24(23):12200-12207

57. Zhao Z, Li L, Du P, Ma L, Zhang W, Zheng L, Lan B, Zhang B, Ma F, Xu B et al (2019) Transcriptional Downregulation of miR-4306 serves as a New Therapeutic Target for Triple Negative Breast Cancer. Theranostics 9(5):1401-1416

58. Yang Y, Luo H, Liu S, Zhang R, Zhu X, Liu M, Hu H, Yang Y, Lv Z, Chen M (2019) Platelet microparticles-containing miR-4306 inhibits human monocytederived macrophages migration through VEGFA/ERK1/2/NF-KB signaling pathways. Clin Exp Hypertens 41(5):481-491

59. Raffone A, Travaglino A, Saccone G, Mascolo M, Insabato L, Mollo A, De Placido G, Zullo F (2019) PAX2 in endometrial carcinogenesis and in differential diagnosis of endometrial hyperplasia: A systematic review and meta-analysis of diagnostic accuracy. Acta Obstet Gynecol Scand 98(3):287-299

60. Rewcastle E, Varhaugvik AE, Gudlaugsson E, Steinbakk A, Skaland I, van Diermen B, Baak JP, Janssen EAM (2018) Assessing the prognostic value of PAX2 and PTEN in endometrial carcinogenesis. Endocr Relat Cancer 25(12):981-991

61. Colina JA, Varughese P, Karthikeyan S, Salvi A, Modi DA, Burdette JE (2020) Reduced PAX2 expression in murine fallopian tube cells enhances estrogen receptor signaling. Carcinogenesis 41(5):646-655 


\section{Tables}

Table1-Characteristics of mRNA and miRNA expression profiles

\begin{tabular}{|lllll|}
\hline Accession & Platform & Group & Samples Characteristcs & Age \\
\hline GSE111974 & GPL17077 & RIF(24) & $\begin{array}{l}\text { Failure of pregnancy in } \geq 3 \text { consecutive IVF cycles with } \geq 1 \text { transfer(s) of good quality embryo } \\
\text { in each cycle }\end{array}$ & $\begin{array}{l}32.76 \\
\pm 2.33\end{array}$ \\
\hline & & Control(24) & Had a history of at least one live birth with no associated comorbidities. \\
\hline GSE71332 & GPL18402 & RIF(7) & Failure of implantation after 3 cycles of IVF or ICSI with no less than 10 good quality embryos & $31.57 \pm 4.50$ \\
\hline & & Control(5) & Got pregnancy after embryo implantation no more than three times & $31 \pm 3.16$ \\
\hline
\end{tabular}

The first dataset is mRNA expression profiles and the second one is miRNA expression profiles.

\section{Table 2-Dual luciferase assay primer}

\begin{tabular}{|c|c|}
\hline $\begin{array}{l}\text { PDPN- } \\
\text { WT- } \\
\text { pmirGLO }\end{array}$ & Cacttgcctggcccactcagaatccacggtgacctctccgcttgccaaaataaccgaaggaaagaccgttcaccagacttggctcctctaaacatttgctgttcaaacatgtttttgaatatacattctataa \\
\hline $\begin{array}{l}\text { PDPN- } \\
\text { MUT- } \\
\text { pmirGLO }\end{array}$ & Cacttgcctggcccactcagaatccacggtgacctctccgettgccaaaataaccgaaggaaagaccgttcaccagacttggctcctctaaacatttgctgttcaaacatgtttttgaatatacattctataa \\
\hline $\begin{array}{l}\text { PAX2- } \\
\text { WT- } \\
\text { pmirGLO }\end{array}$ & gccccgggcggccgaaggccgggccgccccgtcccgccccgtagttgctctttcggtagtggcgatgcgccctgcatgtctcctcacccgtggatcgtgacgactcgaaataacagaaacaaagtcaí \\
\hline $\begin{array}{l}\text { PAX2- } \\
\text { MUT- } \\
\text { pmirGLO }\end{array}$ & Gccccgggcggccgaaggccgggccgccccgtcccgccccgtagttgctctttcggtagtggcgatgcgcctgcatgtctcctcacccgtggatcgtgacgactcgaaataacagaaacaaagtcai \\
\hline
\end{tabular}

Table3-Top 20 nodes of DEGs in PPI network conducted based on 325 DEGs 


\begin{tabular}{|llllll|}
\hline Gene & Degree & Gene & Betweenness & Gene & Closeness \\
\hline AGT & 16 & PTGS2 & 14450.87 & PTGS2 & 0.038592 \\
\hline PTGS2 & 14 & MUC1 & 6803.066 & AGT & 0.038429 \\
\hline TOP2A & 13 & AGT & 6584.209 & VCAM1 & 0.038229 \\
\hline AVP & 13 & VCAM1 & 6000.786 & GJA1 & 0.038185 \\
\hline RRM2 & 12 & CENPU & 5574.618 & GPR68 & 0.038057 \\
\hline GNG4 & 12 & AVP & 5513.234 & MUC1 & 0.038044 \\
\hline CXCL11 & 11 & GJA1 & 5251.475 & KDR & 0.037968 \\
\hline CENPU & 10 & TSC2 & 5165.327 & PROM1 & 0.037924 \\
\hline GPR68 & 10 & PROM1 & 5011.375 & GNG4 & 0.037918 \\
\hline VCAM1 & 10 & KDR & 4198.465 & AVP & 0.03778 \\
\hline MUC1 & 9 & GNG4 & 4130.481 & TSC2 & 0.037755 \\
\hline EXO1 & 9 & PDE6A & 3660.51 & JUP & 0.037673 \\
\hline RAD51AP1 & 9 & ST3GAL6 & 3071.586 & PRKAG2 & 0.037661 \\
\hline POLE2 & 9 & POSTN & 2746.785 & CXCL11 & 0.037661 \\
\hline CDKN3 & 9 & PAX2 & 2703.252 & CENPU & 0.037611 \\
\hline KDR & 9 & ASS1 & 2523.089 & LPAR3 & 0.037611 \\
\hline LPAR3 & 9 & PRKAG2 & 2443.258 & BDKRB1 & 0.037611 \\
\hline PENK & 9 & ABCG1 & 2398.07 & PDPN & 0.037599 \\
\hline GJA1 & 9 & NKX2-1 & 2393.507 & PENK & 0.037574 \\
\hline BDKRB1 & 9 & KLHL13 & 2329.919 & POSTN & 0.037556 \\
\hline
\end{tabular}

Degree: results of degree centrality algorithm; Betweenness: results of betweenness centrality algorithm; Closeness: results of Closeness centrality algorithm.

Table4-Key genes and miRNAs in network

\begin{tabular}{|c|c|c|}
\hline & genes & miRNAs \\
\hline $\begin{array}{l}\text { Up- } \\
\text { regulated }\end{array}$ & \begin{tabular}{l} 
LPAR3 \PAX2 \PDE6A \} $\\
{\text { TSC2 }}$ & 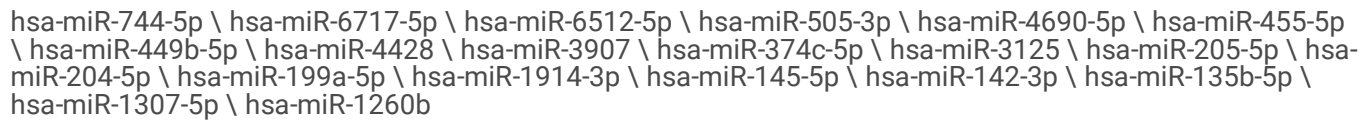 \\
\hline $\begin{array}{l}\text { Down- } \\
\text { regulated }\end{array}$ & $\begin{array}{l}A G T \backslash A S S 1 \backslash G J A 1 \backslash G N G 4 \\
\backslash G P R 68 \backslash K D R \backslash N K X 2-1 \backslash \\
P D P N \backslash P E N K \backslash P R O M 1 \backslash \\
R R M 2 \backslash V C A M 1\end{array}$ & hsa-miR-1207-5p \hsa-miR-4306 \hsa-miR-6088 \hsa-miR-6165 \\
\hline
\end{tabular}
\end{tabular}

\section{Figures}




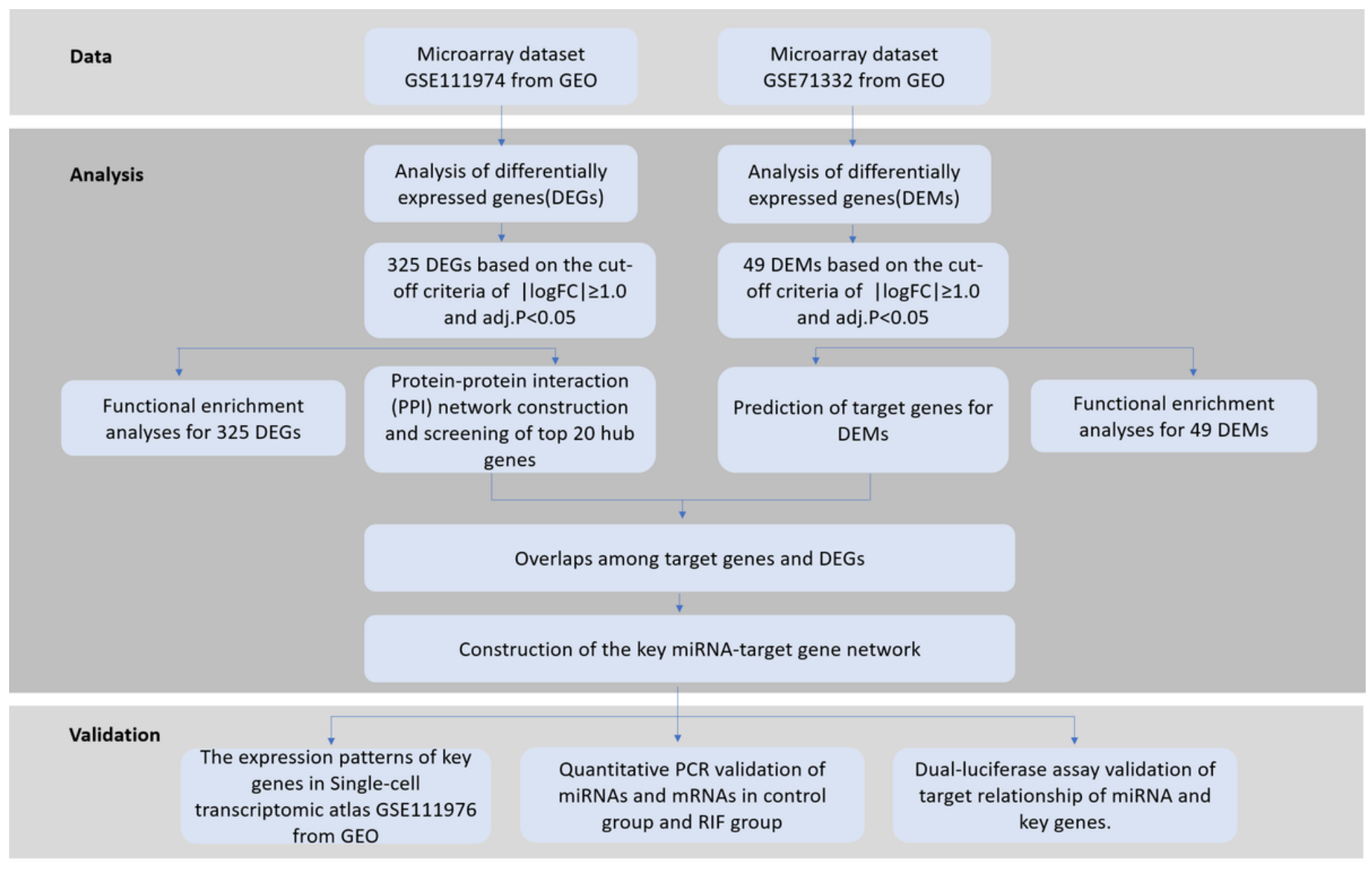

Figure 1

Schematic illustration of this study. 


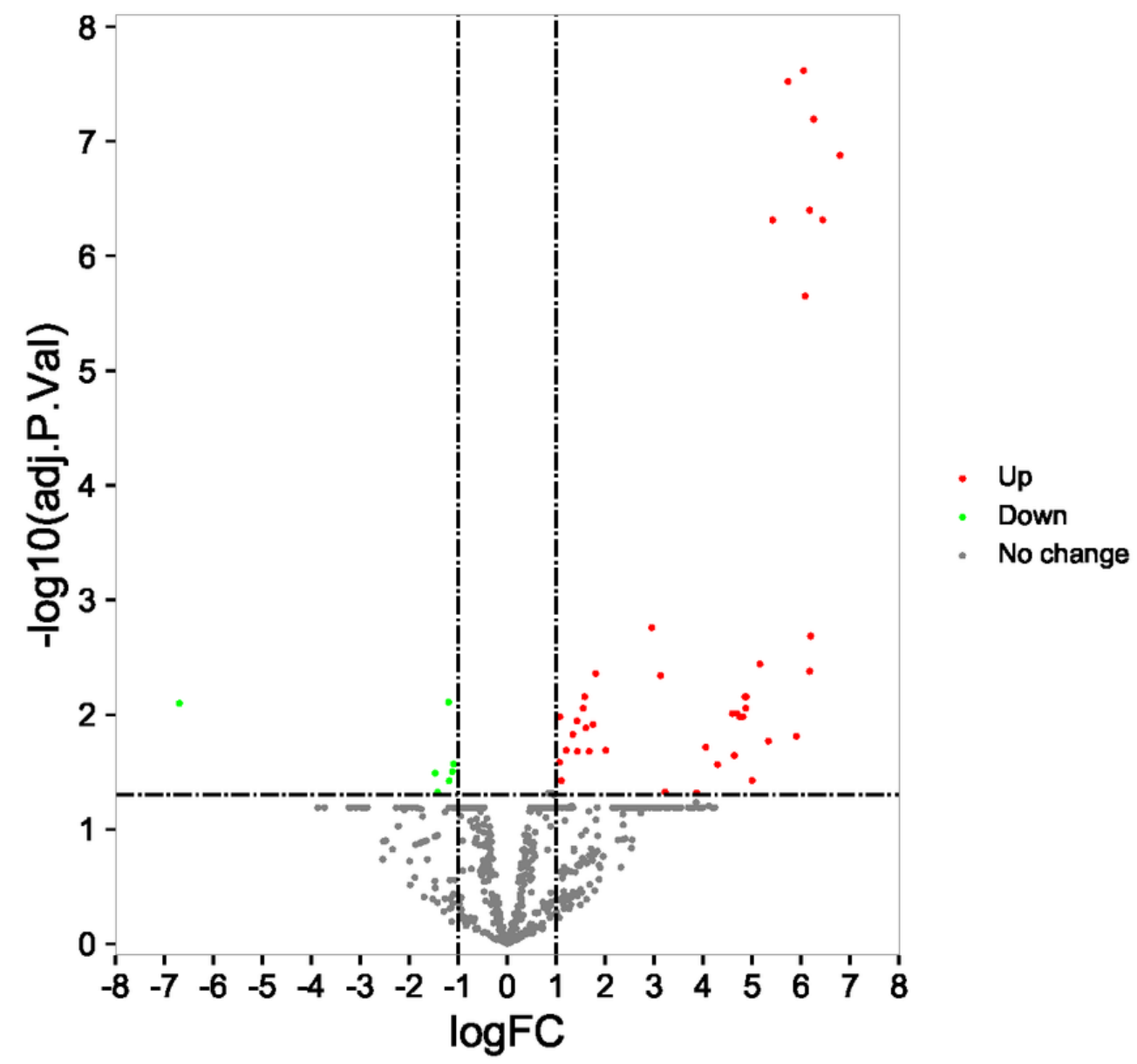

Figure 2

Identification of differentially expressed miRNA in profile datasets.

The 49 differentially expressed miRNAs in GSE71332.

A

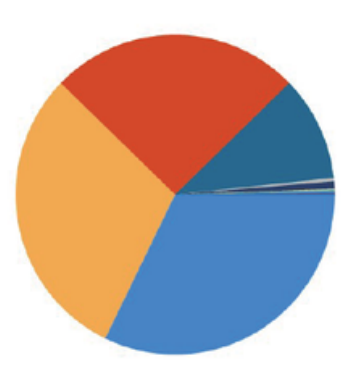

B

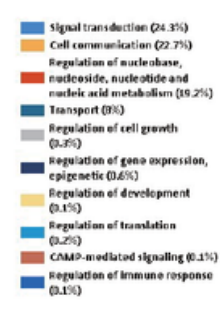

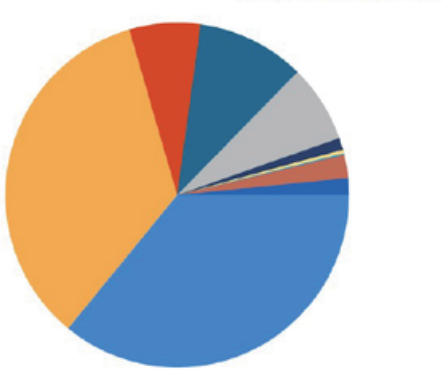

C

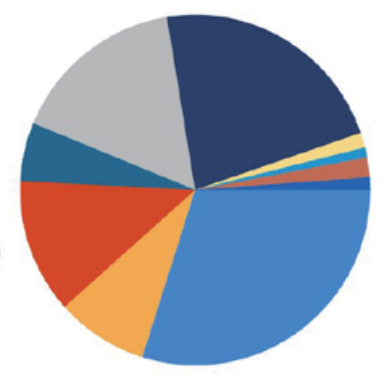

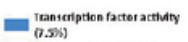
GPase activitr e.1950

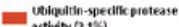
- Grese actio a Gi.s;

Transporter actwity (1.14) Dhosphoric dibstor hydrobse

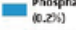
- Provelen serinef/theeasine Konse repulator activity

Figure 3

Functionally enriched GO terms analysis of DEMs in recurrent implantation failure.

BP-associated category, CC-associated category, and MF-associated category of DEMs.

DEMs, differentially expressed miRNAs; GO, Gene Ontology;BP, biological processes; CC, cellular component; MF, molecular function. 


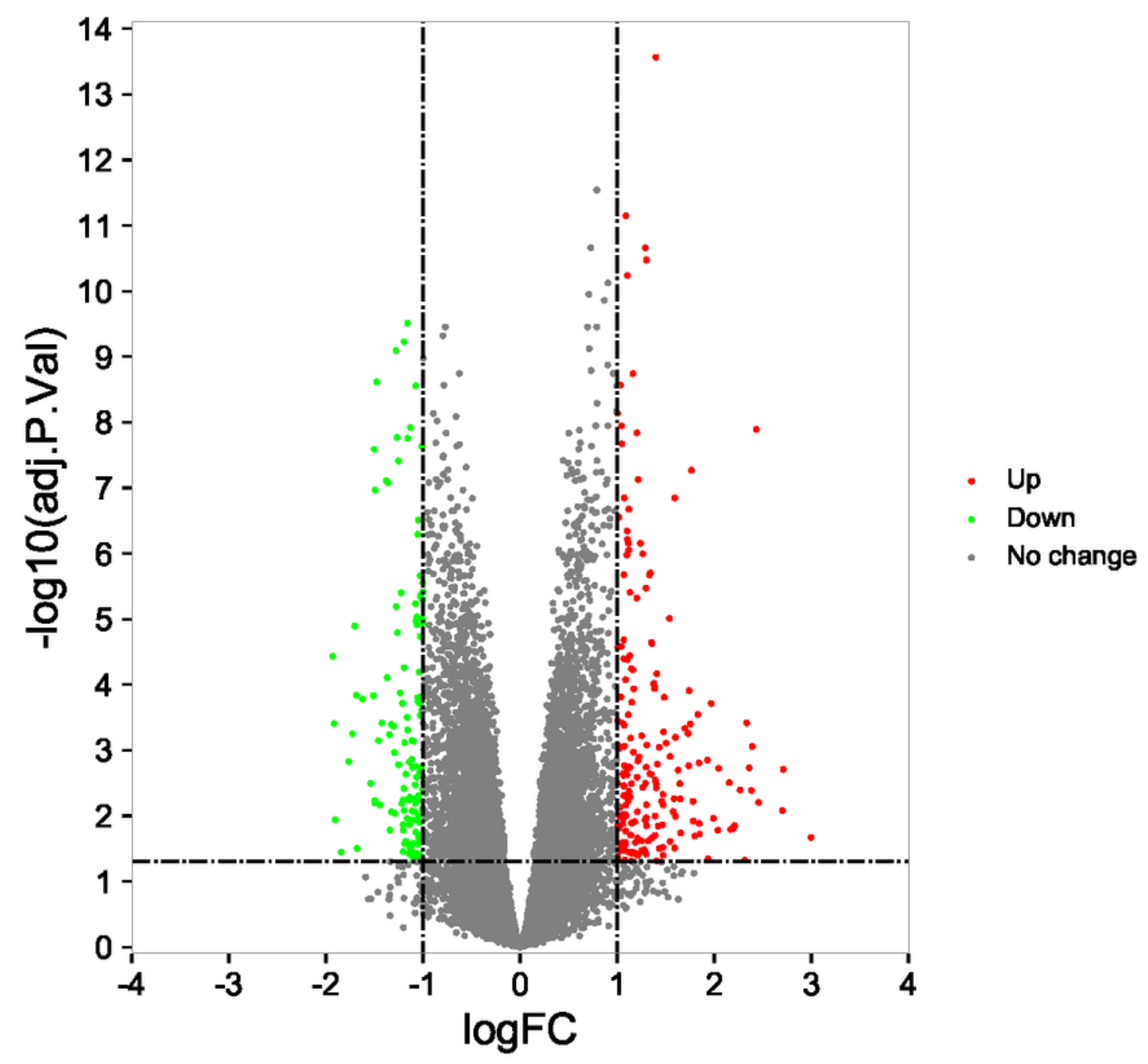

Figure 4

Identification of differentially expressed genes and enrichment analysis

The 325 differentially expressed mRNAs in GSE111974.

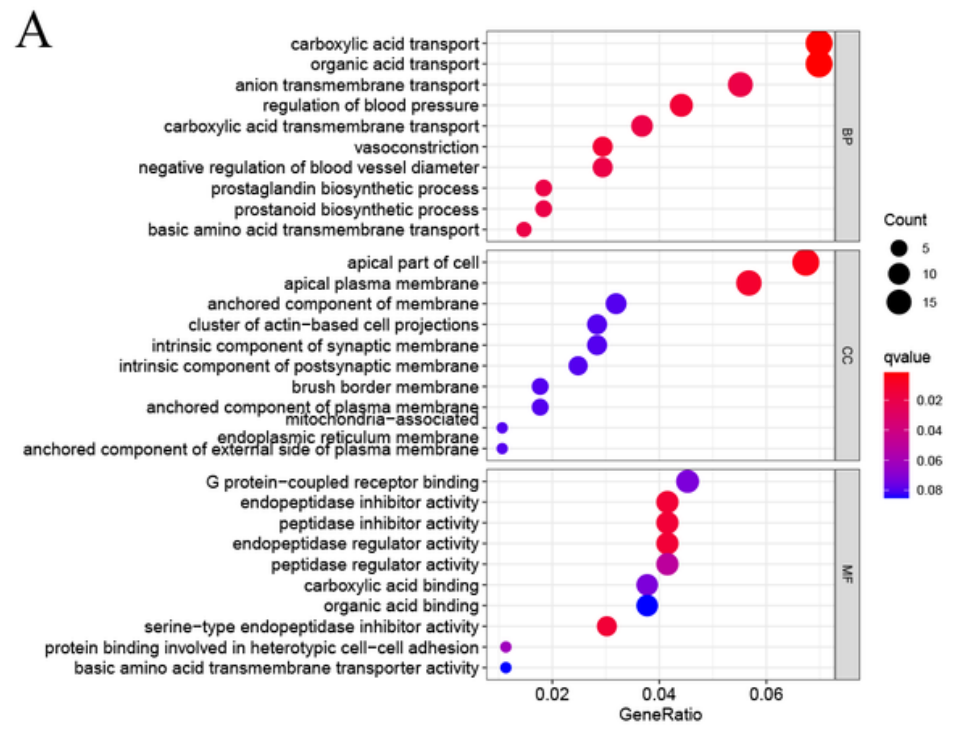

B

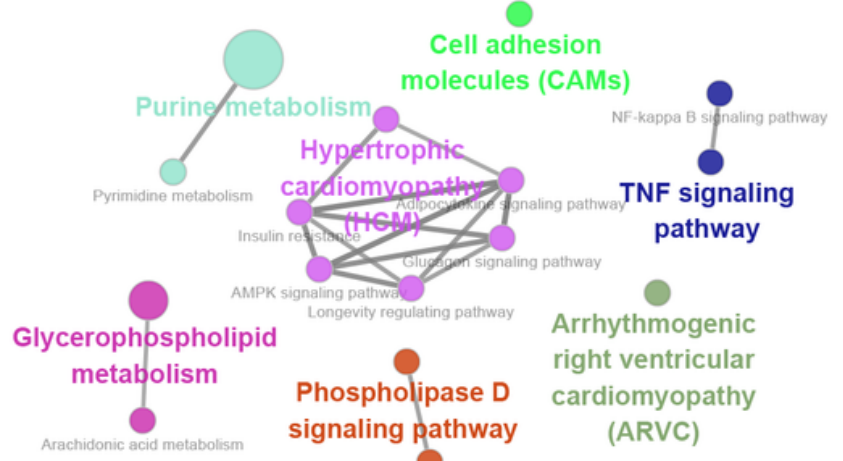

Figure 5 
(A) BP-associated category, CC-associated category, and MF-associated category of DEMs; DEGs, differentially expressed genes; GO, Gene Ontology; (B) KEGG pathways; KEGG, Kyoto Encyclopedia of Genes and Genomes.
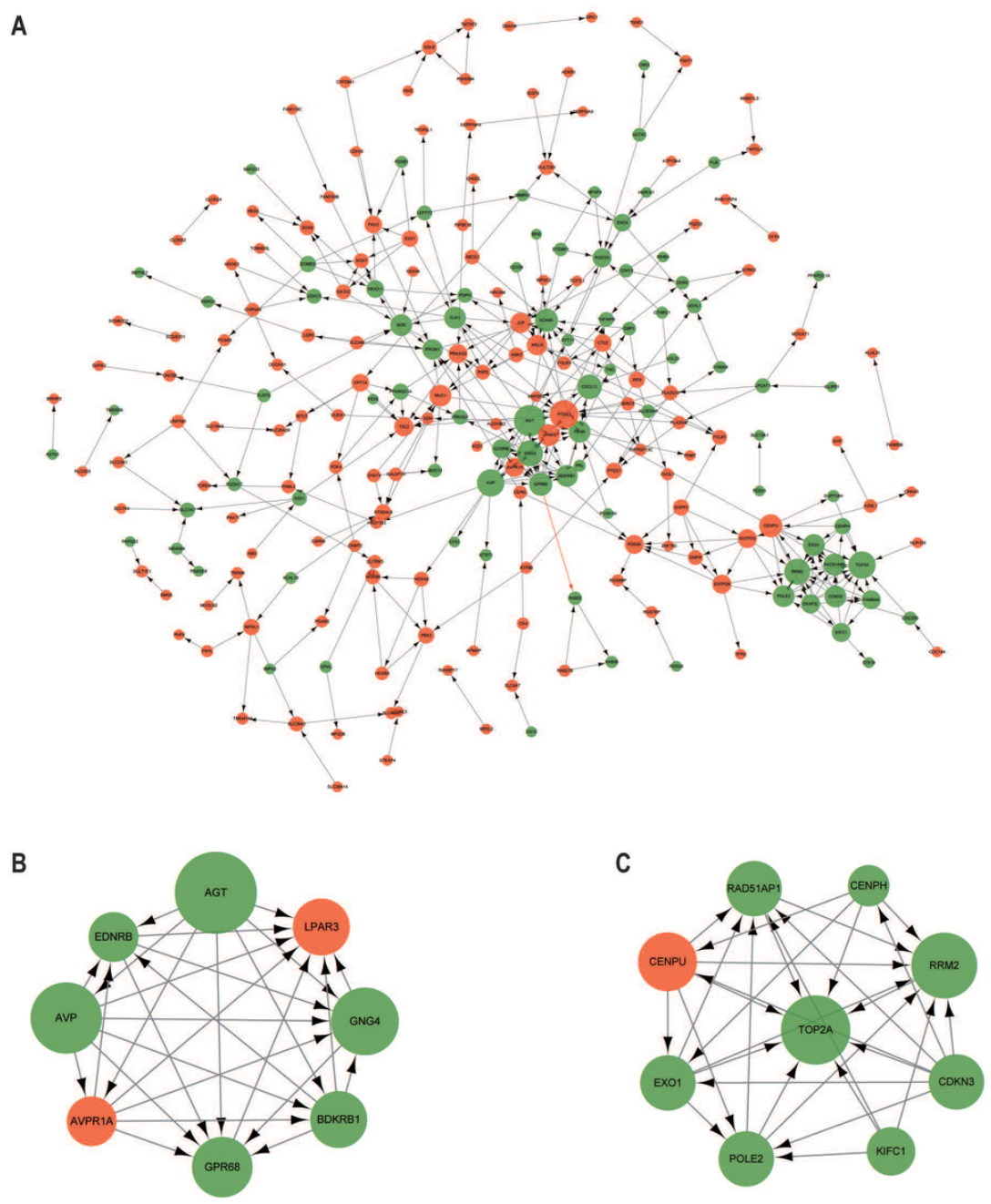

\section{Figure 6}

Protein-protein interaction (PPI) network of DEGs. The size of dot represents the degree of association of the node genes. The larger the dot, the greater the degree of genetic association. Red nodes and green nodes indicate upregulated and downregulated genes, respectively. (A) PPI network contained 229 nodes and 373 interaction pairs; (B) The sub-network module with the highest score in PPI network; (C) A sub-network module with the second highest score in PPI network. 


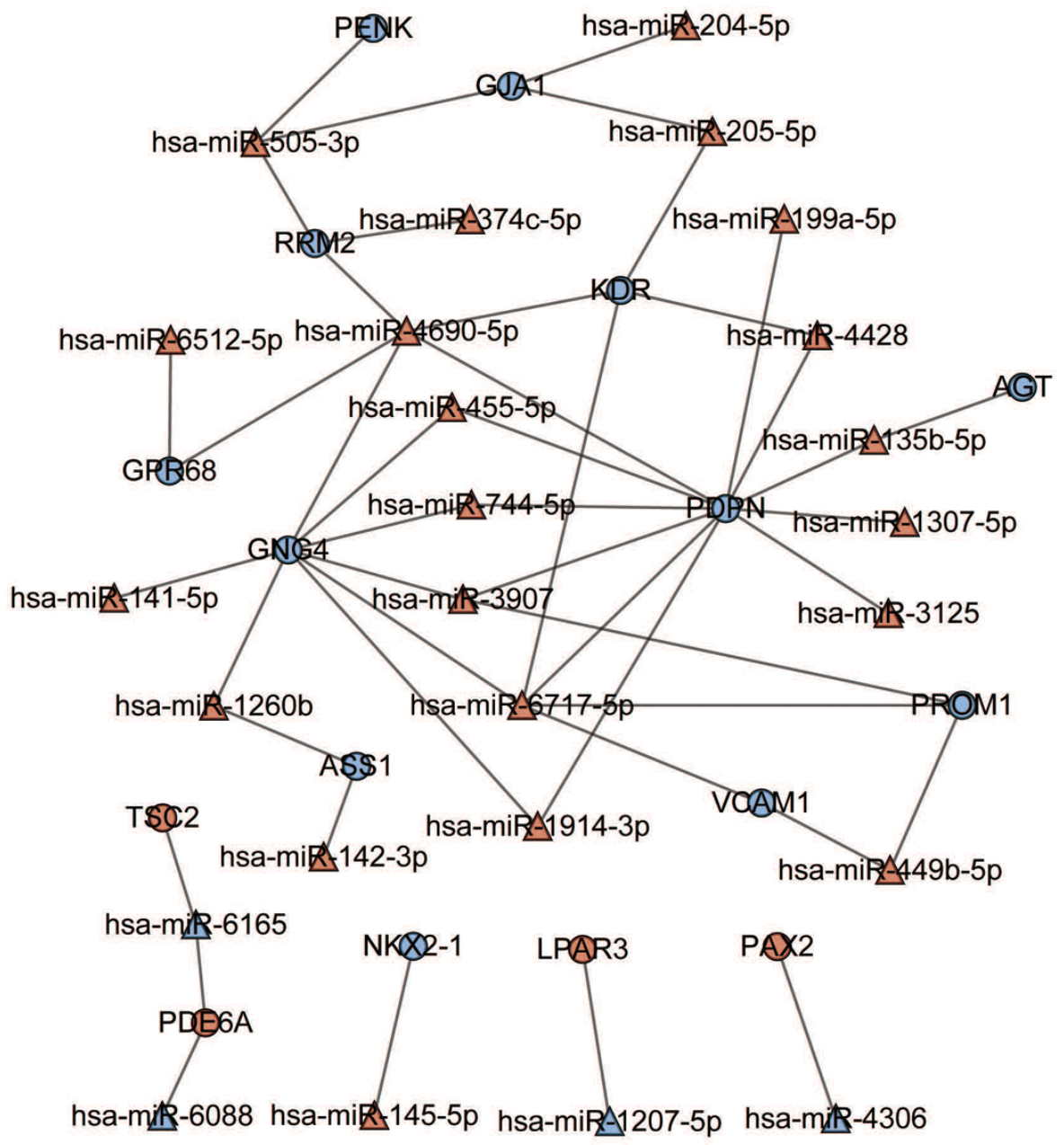

Figure 7

Key miRNA-mRNA network. Triangles represent DEMs and circles represent target DEGs. Red nodes and blue nodes indicate upregulated genes/miRNAs and downregulated genes/miRNAs, respectively. 
$A_{3}$
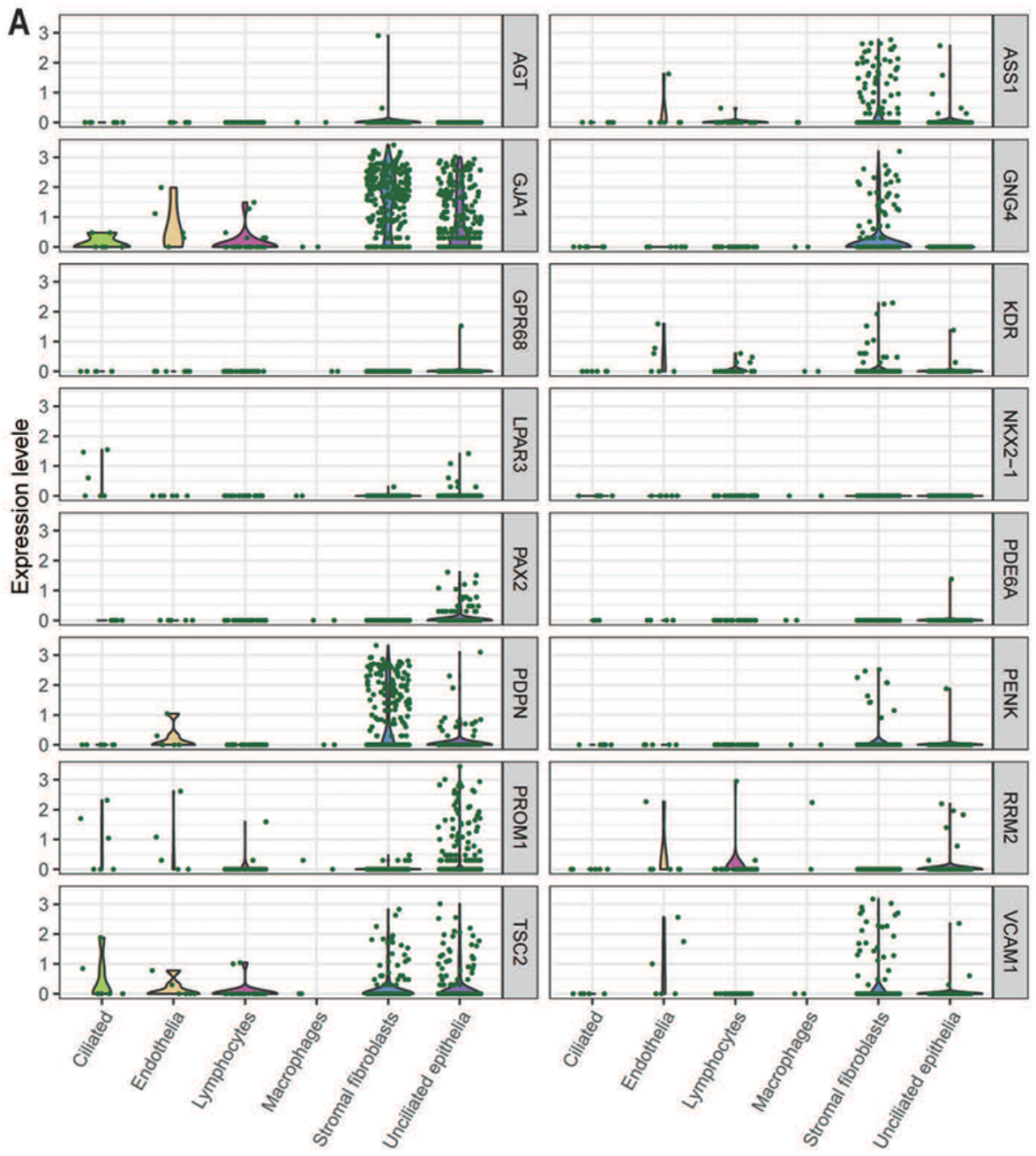
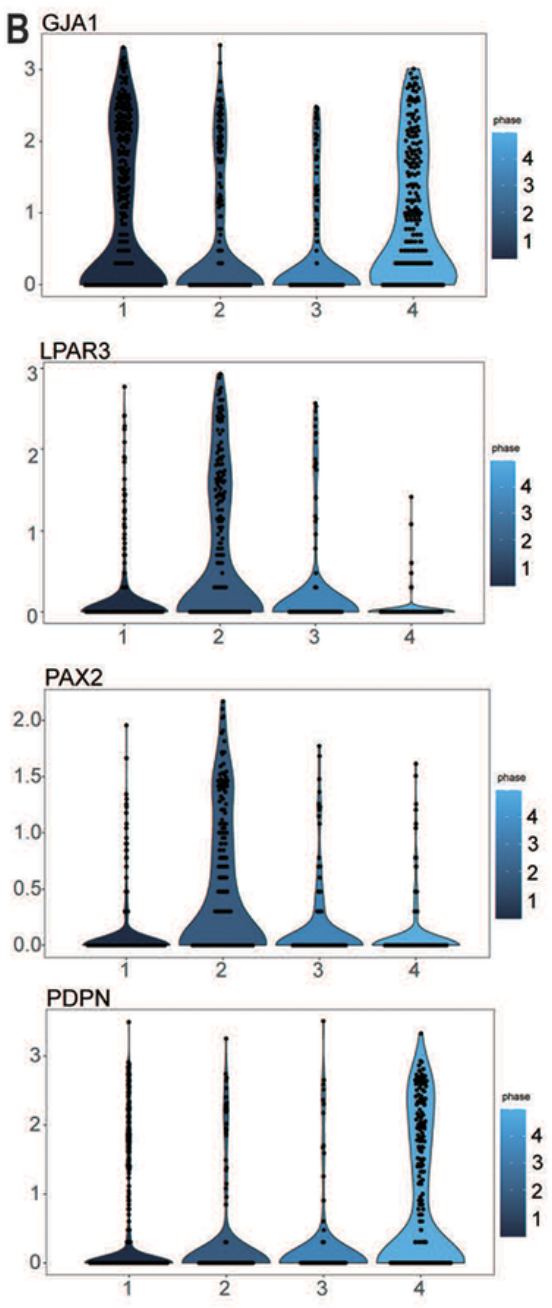

Figure 8

Key genes in Single cell RNA-seq transcriptome profile of normal endometrium. (A) The expression of key genes in mid- and late-secretary menstrual phase. (B) PDPN, GJAT, LPAR3, PAX2 expression variation during four menstrual phases. 

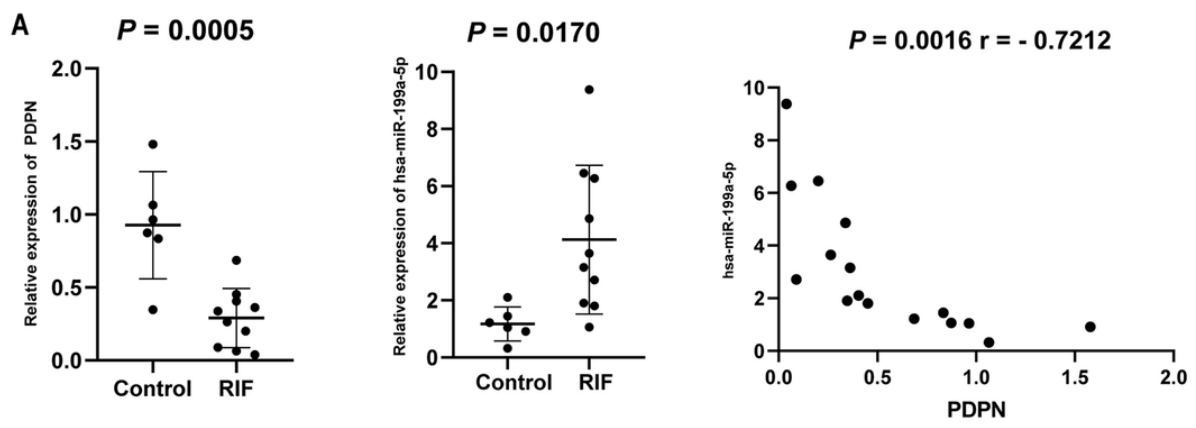

B

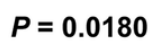

$P=0.0381$
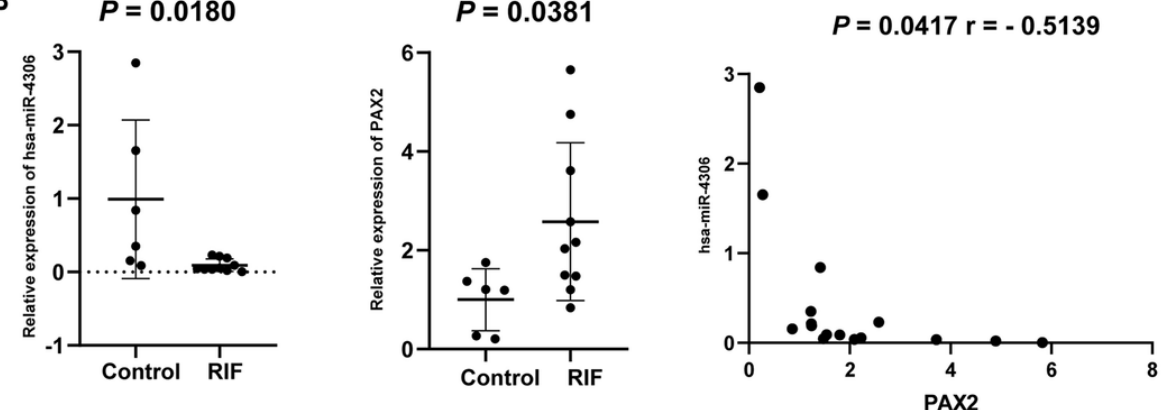

C
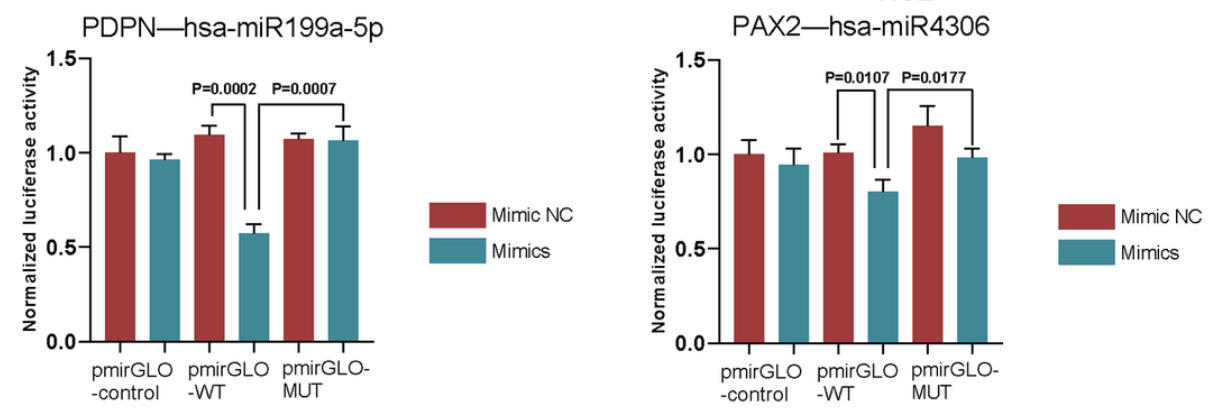

Figure 9

(A-B) Quantitative PCR validation of miRNAs and mRNAs in control group and RIF group. The expression of (A) PDPN and hsa-miR-199a-5p, (B) PAX2 and hsamiR-4306 (C) Dual-luciferase assay validation of target relationship of miRNA and genes. Data are presented as the normalized fold change. Experiments were repeated in triplicate, and values are expressed as mean \pm SEM. * tive. A; miRNA or miR, microRNA. 